\title{
Das Landeskonservatorium der Musik zu Leipzig in der Zeit nach dem Ersten Weltkrieg
}

\author{
Helmut Loos \\ Univerza v Leipzigu \\ Universität Leipzig
}

So tiefgreifend der gesellschaftliche Bruch gewesen ist, den der Erste Weltkrieg ausgelöst hat, so schwer ist zu bestimmen, wie er sich im Einzelnen ausgewirkt hat. Der Wechsel vom Kaiserreich zur Republik stellte eine gesellschaftspolitische Umorientierung dar, die sich auf die Künste dramatisch auswirkte. Der Wegfall der Zensur, die bis dahin vor allem Pornographie und Blasphemie unterdrückt hatte, ermöglichte Werke von Erwin Schulhoffs (1894-1942) Sonata Erotica (1919) und Paul Hindemiths (18951963) Sancta Susanna (1922) bis hin zu Kurt Weills (1900-1950) Dreigroschenoper (1928). Einerseits stellten derartige Werke eine Befreiung bis dahin unterdrückter Emotionen dar, andererseits wurden sie als unerträgliche Provokationen empfunden, die traditionelle Werte nicht nur in Frage stellten, sondern gänzlich zu zerstören drohten. Dabei war das alte Wertesystem keineswegs vollkommen außer Kraft gesetzt, sondern behielt in verschiedenen Bereichen seine Gültigkeit. Die Musik ist dafür ein sprechendes Beispiel. Vor 1914 nicht nur zur höchsten der Künste, sondern geradezu zur Kunstreligion der Moderne von höchstem gesellschaftlichem Rang aufgestiegen, büßte sie ihre Bedeutung keineswegs ein, sondern wahrte ihren Anspruch auf Verkörperung des Wahren, Guten und Schönen, indem sie der überschwänglichen spätromantischen Überwältigungsgeste eine „hei-

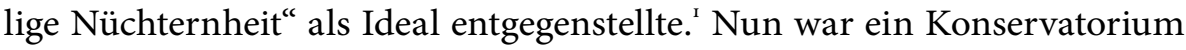

1 Helmut Loos, „Heilige Nüchternheit. Der Komponist in der Moderne. Kontinuität statt Bruch", in Nova glasba $v$ "novi "Evropi med obema svetovnima vojnama - New Music in the "New" Europe Between the Two World Wars, hrsg. von Jernej Weiss (Ko- 
als musikalische Bewahranstalt schon von seinem Namen her eher den traditionellen Werten verschrieben als Neuem aufgeschlossen, und gerade das Königliche Konservatorium der Musik zu Leipzig galt als durch und durch konservativ. Dies entsprach tatsächlich seinem Selbstverständnis, wie aus dem „Personalverzeichnis W. S. 1921-22“ hervorgeht, in dem unter dem Titel „Der Werdegang des Konservatoriums der Musik in Leipzig“ zu lesen ist: „Von Anfang an war damit dem Konservatorium der Geist eines gewissen gesunden Konservativismus mitgegeben worden, den es bis heute nicht verloren hat.“ Und weiter: „daß die Neuromantik, als deren Stammvater Liszt anzuse-


persönlichkeiten im Senat des Konservatoriums bildeten zu dieser Zeit

Professor Stephan Krehl, Studiendirektor [...], Professor Paul Graener, stellvertr. Studiendirektor [...], Professor Otto Lohse, Operndirektor [...], Professor Karl Straube, Thomaskantor [...], Professor Robert Teichmüller [...].

Die Situation in Deutschland nach 1918 darf ohne Übertreibung als katastrophal schlecht beschrieben werden. Eine vernichtende Niederlage und ein bedingungslos angenommener Friedensvertrag von Versailles demütigten eine Nation, die sich zuvor als fortschrittliche Gesellschaft an der Spitze menschlicher Evolution wähnte. Dazu kam eine wirtschaftliche Not, die den Menschen einen täglichen Überlebenskampf aufzwang. Unter diesen bedrückenden Verhältnissen standen alle Institutionen in Frage und mussten sich neu legitimieren, um weiter existieren zu können. Das Leipziger Konservatorium suchte sein Heil in Johann Sebastian Bach, dessen gesellschaftliche Bedeutung in Deutschland bis zum Ersten Weltkrieg beständig zugenommen und einen bis dahin unbekannten Hochstand erreicht hatte. ${ }^{3}$ Karl Straube war an dieser Entwickung seit 1903 als Thomas-

per, Ljubljana: Založba Univerze na Primorskem, Festival Ljubljana, 2018), 51-62. Ich knüpfe hier an mehrere Beiträge an, die ich hier in Ljubljana gehalten habe: „Die kunstreligiöse Botschaft der Leipziger Musikwissenschaft im späten 19. und frühen 20. Jahrhundert", Muzikološki Zbornik 50, Nr. 2 (2014): 43-51. „Synkretismus in der Musik um 1900", in Fin de Siècle and Gustav Mahler $\left(=26^{\text {th }}\right.$ Slovenian Musical Days 2011), hrsg. von Primož Kuret (Ljubljana: Festival Ljubljana, 2012), 73-80.

2 Siehe dazu auch Johannes Forner, „Leipziger Konservatorium und „Leipziger Schule“. Ein Beitrag zur Klassizismus-Diskussion“, Die Musikforschung 50, Nr. 1 (1997): 31-36; Martin Wehnert, Hansachim Schiller und Johannes Forner, Hrsg., Hochschule für Musik Leipzig gegründet als Conservatorium der Musik 1843-1968 (Leipzig: Hochschule für Musik, 1968). 
organist und als Leiter des Leipziger Bachvereins beteiligt. Er gestaltete das 2. Deutsche Bach-Fest in Leipzig 1904 zu einem Aufsehen erregenden Ereignis, er rief eigene Leipziger Bach-Feste ins Leben. 1908 wurde mit einem solchen Fest die Einweihung des neuen Denkmals an der Thomaskirche gefeiert, weitere Leipziger Bach-Feste wurden 1911 und 1914 veranstaltet. Seit 1907 Orgellehrer am Konservatorium und seit 1918 Thomaskantor verfolgte Straube diese Linie in der bedrängenden Nachkriegszeit umso intensiver weiter. Das 8. Deutsche Bach-Fest 1920 in Leipzig stand ebenso unter seiner Leitung wie das Leipziger Bach-Fest 1923. Im Konservatorium gestaltete er am Sonntag, den 23. Oktober 1921, um 12 Uhr vormittags die Eröffnungsfeier des Instituts für Kirchenmusik ${ }^{4}$ ausschließlich mit Bachscher Musik:

1. J. S. Bach, Präludium und Fuge, vorgetragen v. Herrn Günther Ramin, Organist zu St Thomae

2. Begrüßungen und Ansprachen

3. „Jesu meine Freude.“ Motette für 5 Singstimmen von J. S. Bach, vorgetragen vom Thomaner-Chor.'

Selbstverständlich wurde der Thomanerchor von Karl Straube dirigiert, der die Einrichtung des Instituts für Kirchenmusik maßgeblich vorangetrieben hatte und es bis 1948 leitete. Die musikalische Gestaltung der Eröffnungsfeier war Programm und zeigt die überragende Stellung, die Johann Sebastian Bach in der Musik zugeschrieben wurde. Geradezu selbstverständlich teilten die anderen Lehrenden am Konservatorium diese Auffassung, genannt seien nur Günther Ramin (1920-1940), Paul Graener (1920-1924) und Sigfrid Karg-Elert (1919-1933). ${ }^{6}$ Ein einziges Beispiel dafür möge genügen. Als Günther Ramin im Jahre 1973 eine Gedenkschrift zum 75. Geburtstag gewidmet wurde, wählte der Herausgeber Diethard Hell-

Rathert, Kult und Kritik. Aspekte der Bach-Rezeption vor dem Ersten Weltkrieg, 2361.

4 Siehe dazu Maren Goltz, Das Kirchenmusikalische Institut. Spuren einer wechselvollen Geschichte (Leipzig: Hochschule für Musik und Theater 'Felix Mendelssohn Bartholdy", 2001), 20-27.

5 Hochschule Leipzig Archiv, Programmsammlung 1921-1924. Ibid., Leipzig, September 1921: Satzung Institut für Kirchenmusik. Siehe auch C. A. Martienßen, „Das Institut für Kirchenmusik an der Hochschule für Musik in Leipzig“, Zeitschrift für Musik 89 (1922): 102-105.

6 Hochschule Leipzig Archiv, Karg-Elert, Siegfried: Die „Übersicht über die von KargElert 1928 geprüften Studenten“ enthält unter dem Datum vom 4. März 1896 einen weiteren litauischen Studenten, Kasimir Banaitis aus Woitiekapiai (Inskription Nr. 14056). 
mann als Titel des Buches die verkürzte Überschrift eines Vortrags, den Ramin 1954 an der Universität Zürich gehalten hatte: „Johann Sebastian Bach. Ende und Anfang“. Der originale Titel hatte gelautet: „Johann Sebastian Bach als Ende und Anfang und seine Bedeutung für die geistige Entwicklung der Jugend".7 Ramin stellte ganz bewusst das berühmte Diktum eines früheren, berühmten Konservatoriumlehrers, Max Regers, von Bach als „Anfang und Ende aller Musik“ um. Ein anderer Titel eines bis dahin unveröffentlichten Beitrags von Ramin trägt die Überschrift „Bachs Totalität in Werk und Wesen“. Die Wortwahl verweist direkt in den Bereich des Totalitarismus des Dritten Reiches, in die gerade die hier geschilderte Leipziger Schule sich vielfach verstrickt hat. In teilweise erregten Auseinandersetzungen ist dies in den letzten Jahrzehnten aufgearbeitet worden. ${ }^{8}$

Die wirtschaftlichen Probleme des Konservatoriums als privater Stiftung Leipziger Bürger, vor denen sich die Bach-Pflege in der Zwischenkriegszeit vollzog, waren erheblich und haben sich auf die Arbeit und auf das Selbstverständnis des Konservatoriums ausgewirkt. Die finanzielle Lage des Konservatoriums und seiner Beschäftigten war ausgesprochen schlecht. Die Zahl der Schüler hatte sich im Ersten Weltkrieg dramatisch verringert, da viele Studierende zum Kriegsdienst eingezogen und Ausländer verfeindeter Staaten vom Studium ausgeschlossen worden waren. Die Erträge aus dem Stiftungsvermögen waren infolge der Inflation eingebrochen und brachten kaum noch Gewinn. Zuschüsse aus öffentlicher Hand mussten in Anspruch genommen werden, waren aber knapp bemessen und boten keine längerfristig verlässliche Grundlage, da eine angestrebte Verstaatlichung scheiterte. ${ }^{9}$ Die Kunde von der Gründung einer staatlichen Hochschule für Musik in Dresden musste unter solchen Umständen den entsetzten Widerspruch des Leipziger Konservatoriums hervorrufen. ${ }^{10}$ Am 12. März 1920 erhob das Konservatorium beim Ministerium für Kultur und

7 Diethard Hellmann, Hrsg., Johann Sebastian Bach. Ende und Anfang. Gedenkschrift zum 75. Geburtstag des Thomaskantors Günther Ramin (Wiesbaden: Breitkopf und Härtel, 1973).

8 Hans-Joachim Schulze, Ulrich Leisinger und Peter Wollny, Hrsg., Passionsmusiken im Umfeld Johann Sebastian Bachs. Bach unter den Diktaturen 1933-1945 und 19451989. Bericht über die Wissenschaftliche Konferenz anlässlich des 69. Bach-Festes der Neuen Bachgesellschaft Leipzig, 29. und 3o. März 1994 (Hildesheim, Zürich und New York: Olms, 1995). die Staatliche Hochschule für Musik Leipzig in der Zeit des Nationalsozialismus 19331945 (Stuttgart: Franz Steiner Verlag, 2013), 39-45. 
öffentlichen Unterricht in Dresden in einem ausführlichen Brief Einspruch und forderte unter ausführlicher Darlegung des Stiftungsvermögens einen staatlichen Zuschuss für das eigene Haus. ${ }^{\text {II }}$ Das Leipziger Tageblatt und Handels-Zeitung meldete am Mittwoch, den 16. März 1921:

Wie von Regierungsseite aus mitgeteilt wird, plant die sächsische Regierung, das Konservatorium und die Frauenberufsschule in Leipzig auf den Staat zu übernehmen. ${ }^{\text {I2 }}$

Doch trotz einer Neustrukturierung des Leipziger Konservatoriums mit einer neuen Verfassung am 1. April 1921 verschlimmerte sich die Lage weiter. ${ }^{13}$ Im Jahre 1922 wurde das Unterrichtsgeld um 300 \% erhöht, gleichzeitig verhandelte das Kuratorium ernsthaft über die Auflösung des Konservatoriums.

Wie verzweifelt die Lage sich darstellte, geht aus Beiträgen im 1. Märzheft 1922 der Zeitschrift für Musik hervor, erschienen in Leipzig am Sonnabend, den 4. März, unter dem Hauptschriftleiter Dr. Alfred Heuß. Eingangs philosophiert Franciscus Nagler hier über Messe und Musik, über die kirchenmusikalische Gattung und den Markt (wirtschaftlichen Handel) in ihrem historischen Zusammenhang, um zu den Messeteilen Kyrie, Gloria, Credo, Sanctus, Benedictus, Agnus Dei aktuelle Texte im Tenor flehender Bitten zu formulieren:

Erbarmen! Erbarmen! Die musikalische Not unseres Volkes ist groß. Es schreit nach Brot, und man bietet ihm Steine. Der Gassenhauer triumphiert. Die Masse trottelt fremden Schelmen nach. Mengen von Gebildeten hinterher. Vox populio - foxtrott! [...] Wir glauben an die unvergängliche Kraft deutscher Musik. ${ }^{14}$

In einem „Aufruf zur Hilfeleistung für das Konservatorium für Musik zu Leipzig“ beklagt Stephan Krehl, dass die Bezüge der Konservatoriumslehrer nicht einmal die Höhe der Proletariergehälter erreichten. ${ }^{\text {Is }}$ Leiden-

11 Hochschule Leipzig Archiv, HfM Jg. 1920: „Konservatorium der Musik zu Leipzig / An das Ministerium des Kultur und öffentlichen Unterrichts Dresden / Leipzig, den 12. März 1920".

12 Hochschule Leipzig Archiv, HfM Jg. 1921: „Leipziger Tageblatt und Handels-Zeitung. Zweite Abend-Ausgabe 115. Jahrgang. 1921. Mittwoch, den 16. März. Geplante Verstaatlichung des Konservatoriums und der Frauenberufsschule in Leipzig“.

13 Hochschule Leipzig Archiv, HfM Jg. 1921: „Leipzig, den 23. März 1921. Ankündigung einer neuen Verfassung, Handzettel für Lehrer und Lehrerinnen“.

14 Franciscus Nagler, „Messe und Musik“, Zeitschrift für Musik 89 (1922): 97-99.

15 Zeitschrift für Musik 89 (1922): 100. 
schaftlich plädiert er gegen die Pläne der Gründung einer neuen Hochschule für Musik in Dresden, auch wenn sie einstweilen vom Landtag abgelehnt worden sei, und für eine Stärkung der eigenen Einrichtung.

Die Studierenden bildeten eine „Vereinigung der Studierenden am Konservatorium zu Leipzig“, um die größten Notlagen gemeinsam zu bewältigen. Die „Vereinigung der Freunde und Förderer des Konservatoriums der Musik zu Leipzig“" unterstützte den Spendenaufruf ganz praktisch und ein wenig hilflos mit der Angabe ihrer Bankverbindung. ${ }^{16}$ Nur durch einen Zuschuss der Stadt Leipzig von 2 1/2 Millionen Mark wurde die Schließung abgewendet, doch war dies keine Lösung auf Dauer, vielmehr richtete sich weiter alle Hoffnung auf den Staat. ${ }^{17}$ Als Stephan Krehl am 9. April 1924 verstarb, war die Lage keineswegs gesichert, Walter Davisson führte vertretungsweise die Direktorialgeschäfte weiter bis zur Berufung des neuen Direktors Max Pauer ${ }^{18}$ Die eingeleiteten Maßnahmen waren drastisch: trotz weiterer Unterstützung durch die Sächsische Regierung wurden Lehrkräfte gekündigt, das Haus des Konservatoriums als „Musik-Meßhaus" vermietet und sogar der wertvollste Teil der Bibliothek verkauft, er wurde der Vereinigung der Freunde der Universität zugunsten des Musikwissenschaftlichen Instituts zugeschlagen (heute in den Sondersammlungen der Bibliotheca Albertina). Anscheinend waren diese Maßnahmen und die Verhandlungen des neuen Direktors relativ erfolgreich, denn die Leipziger Neuesten Nachrichten vermeldeten am Donnerstag, den 5. Juni 1924, unter dem Titel „Die Gesundung des Leipziger Konservatoriums. Der neue Direktor", dass

Max Pauer den an ihn ergangenen Ruf als Studiendirektor der Anstalt angenommen "habe, weil „nunmehr die finanzielle Sanierung des Instituts durchgeführt ist und damit das Konservatorium wieder auf gesicherter wirtschaftlicher Grundlage steht. ${ }^{19}$

Unterstützt wurden diese Maßnahmen von einem neuen Kuratorium (früher Direktorium), dem ein erfahrener Geschäftsmann wie Henri

18 Hochschule Leipzig Archiv, HfM Jg. 1924: „Leipziger Abendpost. Dienstag, den 27. Mai 1924“. Siehe auch Leipziger Abendpost. Dienstag, den 27. Mai 1924: „Krehl-Feier im Konservatorium. Die musikalische Gedächtnisfeier für den unlängst verstorbenen Studiendirektor der Anstalt, Stephan Krehl“.

19 Hochschule Leipzig Archiv, HfM Jg. 1924: „Leipziger Neueste Nachrichten. Donnerstag, den 5. Juni 1924“. 
Hinrichsen nur unter größten Bedenken beitrat. Die finanziellen Geschäfte führte nun der Unternehmer Gustav Flinsch, der eine Stabilisierung der Lage erreichte. Die verschiedenen Reformbemühungen hatten insofern Erfolg, als 1927 die staatliche Anerkennung als Landeskonservatorium für Musik zu Leipzig gelang, die auch mit finanzieller Absicherung verbunden war. 1930, als der neue Oberbürgermeister Carl Goerdeler den Vorsitz des Kuratoriums übernahm und die verwaltungstechnischen Angelegenheiten professionell ordnete, trat Flinsch von seinen Posten zurück (er wurde wegen Veruntreuung verklagt).

Die Krisen des Konservatoriums, die hier nur angedeutet werden können, sind erst in jüngster Zeit musikwissenschaftlich thematisiert worden. ${ }^{20}$ $\mathrm{Zu}$ lange hat eine Glorifizierung vorgeherrscht, die derartige Vorgänge nicht realistisch darzustellen vermochte. Dass sie sich nicht auf das Selbstverständnis des Konservatoriums ausgewirkt hätten, erscheint höchst unwahrscheinlich. Dabei war die Situation in Leipzig durchaus kein Einzelfall, sondern nur eine Facette der krisenhaften Situation in ganz Deutschland. Vergleicht man unter diesen Prämissen einmal die beiden Bach-Umfragen, die von der Zeitschrift Die Musik $1905^{21}$ und (weniger umfangreich) 1930 veröffentlicht worden waren, so fällt auf, dass auf den 73 Seiten der ersten Umfrage nahezu ausschließlich ${ }^{22}$ emphatische Bekenntnisse zu Bach als nationalen Heroen und Kraftquell deutschen Geistes zu finden sind (berühmt ist Max Regers Beitrag). 1930 ist einerseits eine Steigerung der Emphase zu verzeichnen, etwa von Waldemar von Bausznern: „Er ist für mich das Unermeßliche " und Joseph Haas: „der Inbegriff alles Höchsten“, ${ }^{23}$ andererseits

20 Yvonne Wasserloos, Das Leipziger Konservatorium der Musik im 19. Jahrhundert. Anziehung und Ausstrahlungskraft eines musikpädagogischen Modells auf das internationale Musikleben (Hildesheim, Zürich und New York: Olms, 2004); Stefan Keym, Symphonie-Kulturtransfer. Untersuchungen zum Studienaufenthalt polnischer Komponisten in Deutschland und zu ihrer Auseinandersetzung mit der symphonischen Tradition 1867-1918 (Hildesheim: Olms, 2010); Stefan Keym, „Leipzig oder Berlin? Statistik und Ortswahlkriterien ausländischer Kompositionsstudenten um 1900 als Beispiel für einen institutionsgeschichtlichen Städtevergleich“, in $\mathrm{Mu}$ sik in Leipzig, Wien und anderen Städten im 19. Und 20. Jahrhundert: Verlage - Konservatorien - Salons - Vereine - Konzerte (Musik - Stadt. Traditionen und Perspektiven urbaner Musikkulturen, Bd. 3), Hrsg. Stefan Keym und Katrin Stöck (Leipzig: Gudrun Schröder Verlag, 2011), 142-164.

21 Anon., „Was ist mir Johann Sebastian Bach und was bedeutet er für unsere Zeit?", Die Musik 5, Bd. 17 (1905/06): 6-78.

22 Ausnahmen bilden etwa Oskar Sonneck, Hans Huber, Arnold Mendelssohn und Georg Schumann, deren kritische Bemerkungen sich allerdings vor allem auf aufführungspraktische Fragen beziehen. 
sind aber distanziertere Bach-Deutungen zu finden. So schreibt Kurt Weill, Bachs Werk sei „von höchster Zweckmäßigkeit. Deswegen (nicht trotzdem) bewahrt es ein einzigartiges Niveau. ". ${ }^{24}$ Auch die Kirchenmusikalische Erneuerungsbewegung befleißigte sich eher „heiliger Nüchternheit“. Gemeinsam war den unterschiedlichen Gewichtungen das Bild von Bach als Urvaters der deutschen Musik, wie es sich seit Nikolaus Forkels Bach-Buch aus dem Jahre 1802 herausgebildet hatte. In der Notsituation nach dem Ersten Weltkrieg erhielt es eine geradezu apologetische Überhöhung, die deutsche Musik und ihr Urvater Bach erschienen als Retter der Nation aus der Katastrophe. In diesem Sinne waren Bach-Feste weniger kulturelle Ereignisse als nationale Kundgebungen mit religiösem Anspruch, Leipzig mit seinem Konservatorium bildete dafür einen Kristallisationspunkt.

Denn gerade die in Leipzig erscheinenden Musikzeitungen verbreiteten dieses Bild über Europa hinaus. In der Zwischenkriegszeit geschah dies vor dem düsteren Panorama äußerer und innerer Not. Georg Göhler, ${ }^{25} \mathrm{Ab}-$ solvent des Leipziger Konservatoriums und Leipzig u. a. als Autor der (Neuen) Zeitschrift für Musik vielfach verbunden, stellte einen direkten Zusammenhang her: „Die innere Ursache der Notlage der deutschen Musik ist der sittliche Zustand des Volkes, die herrschende Lebensauffassung. ${ }^{226}$ Nicht geringen Schaden habe die Überschätzung von Richard Strauss verursacht, die inneren Ursachen der Notlage aber müssten überwunden werden durch sittliche Erneuerung, die „äußeren Ursachen [würden] einzig und allein getilgt durch die Beseitigung des Friedensvertrages von Versailles." Und nochmals erwähnt er den tief sitzenden Stachel, dem das Unheil zugeschrieben wurde (man wagt kaum zu fragen, was dies mit Musik zu tun habe):

Ist das deutsche Volk unfähig zur sittlichen Erneuerung und zur Einigkeit, die die Revision des Versailler Vertrages erzwingt, dann hat auch für die deutsche Musik als Weltmacht die letzte Stunde geschlagen. ${ }^{27}$

Im gleichen Heft der Zeitschrift für Musik ruft Rudolf Cahn-Speyer dazu auf, die Notlage der Musiker durch Beitritt zum Verband konzertierender

24 Ibid., 267.

25 Zur Person Georg Göhler aus zeitgenössischer Sicht siehe Zeitschrift für Musik 88 (1921): 495 .

26 Georg Göhler, „Die Notlage der deutschen Musik“, Zeitschrift für Musik 88 (1921): 487. 
Künstler zu lindern. ${ }^{28}$ Die Rolle der Musikfeste streicht Alfred Heuß hervor, wenn er über das zehnte Deutsche Bachfest in Breslau vom 7. bis 9. Oktober 1922 schreibt,

daß alle diese Feste mehr als nur gewöhnliche Musikfeste sind, weil jeder denkende Besucher unwillkürlich fühlt, daß sie sich auf Grundlagen deutschen Wesens aufbauen, die zu unserer Erstarkung dringend notwendig sind. ${ }^{29}$

Im gleichen Tenor berichtet T. Niechciol über die Feier des hundertjährigen Bestehens der Staatlichen Akademie für Schul- und Kirchenmusik in Berlin:

Es war kein Fest in des Wortes profaner Bedeutung, sondern ein Erleben, eine machtvolle Kundgebung deutscher Kunst und deutschen Empfindens; ein Sonnenstrahl in das kranke deutsche Herz und ein hoffnungsvoller Ausblick. ${ }^{30}$

Dass die Beschwörung des Nationalen in der Musik kaum mehr Grenzen kannte, geht aus dem Bericht von 1923 hervor, dass Henri Marteau bei einem Konzert in Deutschland am Auftreten gehindert und als Franzose vertrieben worden sei, weil er für "deutschen Wesens durchaus unwürdig“ gehalten worden sei. Zwar beteuert die Schriftleitung, dass solche Ausschreitungen zu verurteilen man "auch in streng nationalen Kreisen durchaus einig“ sei, aber:

Wer garantiert uns nun dafür, daß das Verfahren in München nicht bei jüdischen Musikern und Dirigenten wiederholt wird? Die Sturmtruppen Hitlers wollen Taten verrichten. ${ }^{31}$

Dem Einfluss des Nationalsozialismus hat das Leipziger Konservatorium nicht erst ab 1933 keinen Widerstand geleistet, sondern Dozentenschaft und Studentenschaft sind seiner Ideologie in breiter Front willig gefolgt. Dies entsprach der allgemeinen Tendenz des deutschen Musiklebens und stellte keinen Einzelfall dar. Die Folgen wurden erst lange nach dem Zweiten Weltkrieg sichtbar, als der moralische Anspruch der Ernsten Mu-

28 Rudolf Cahn-Speyer, „Die Notlage der konzertierenden Künstler“, Zeitschrift für Musik 88 (1921): 492-495, 515-517.

29 Alfred Heuß, „Das zehnte Deutsche Bachfest in Breslau 7. bis 9. Oktober 1922“, Zeitschrift für Musik 89 (1922): 481.

30 T. Niechciol, „Die Feier des hundertjährigen Bestehens der 'Staatlichen Akademie für Schul- und Kirchenmusik' in Berlin“, Zeitschrift für Musik 89 (1922): 300-302.

31 J. E. Robert, „Bach, Beethoven und Stinkbomben“, Zeitschrift für Musik 9o (1923): 6. 
sik als Kunstreligion der Moderne zu schwinden begann. Die gesellschaftliche Ausnahmestellung der Musik und ihr Anspruch als moralische Instanz gingen verloren, als die ungeheuren Verbrechen des Dritten Reichs nach und nach ins kollektive Bewusstsein drangen, die sie nicht verhindert, sondern eher noch befördert hatte.

\section{Bibliographie}

Anon. „Bekenntnisse zu Bach“. Die Musik XXII, Bd. 4 (Januar 1930): 265-267.

Anon. „Was ist mir Johann Sebastian Bach und was bedeutet er für unsere Zeit?“. Die Musik 5, Bd. 17 (1905/06): 3-78.

Cahn-Speyer, Rudolf. „Die Notlage der konzertierenden Künstler“. Zeitschrift für Musik 88 (1921): 492-495, 515-517.

Forner, Johannes. „Leipziger Konservatorium und „Leipziger Schule“. Ein Beitrag zur Klassizismus-Diskussion“. Die Musikforschung 50, Nr. 1 (1997): 31-36.

Göhler, Georg. „Die Notlage der deutschen Musik“. Zeitschrift für Musik 88 (1921): 487-489.

Goltz, Maren. Das Kirchenmusikalische Institut. Spuren einer wechselvollen Geschichte. Leipzig: Hochschule für Musik und Theater 'Felix Mendelssohn Bartholdy“, 2001.

Goltz, Maren. Musikstudium in der Diktatur. Das Landeskonservatorium der Musik / die Staatliche Hochschule für Musik Leipzig in der Zeit des Nationalsozialismus 1933-1945. Stuttgart: Franz Steiner Verlag, 2013.

Heinemann, Michael und Hans-Joachim Hinrichsen, Hrsg. Bach und die Nachwelt, Bd. 3: 1900-1950. Laaber: Laaber Verlag, 2000.

Hellmann, Diethard, Hrsg. Johann Sebastian Bach. Ende und Anfang. Gedenkschrift zum 75. Geburtstag des Thomaskantors Günther Ramin. Wiesbaden: Breitkopf und Härtel, 1973.

Heuß, Alfred. „Das zehnte Deutsche Bachfest in Breslau 7. bis 9. Oktober 1922“. Zeitschrift für Musik 89 (1922): 481-483.

Hochschule leipzig Archiv. 1920-1928. Leipzig.

Keym, Stefan. Symphonie-Kulturtransfer. Untersuchungen zum Studienaufenthalt polnischer Komponisten in Deutschland und zu ihrer Auseinandersetzung mit der symphonischen Tradition 1867-1918. Hildesheim: Olms, 2010.

Keym, Stefan. „Leipzig oder Berlin? Statistik und Ortswahlkriterien ausländischer Kompositionsstudenten um 1900 als Beispiel für einen institutionsgeschichtlichen Städtevergleich “. In Musik in Leipzig, Wien und anderen Städten im 19. Und 20. Jahrhundert: Verlage - Konservatorien - Salons - Vereine - Konzerte (Musik - Stadt. Traditionen und Perspektiven urbaner Musikkulturen, Bd. 
3), hrsg. von Stefan Keym und Katrin Stöck, 142-164. Leipzig: Gudrun Schröder Verlag, 2011.

Loos, Helmut. „Synkretismus in der Musik um 1900“. In Fin de Siècle and Gustav Mahler (=26 $6^{\text {th }}$ Slovenian Musical Days 2011), hrsg. von Primož Kuret, 73-80. Ljubljana: Festival Ljubljana, 2012.

Loos, Helmut. „Die kunstreligiöse Botschaft der Leipziger Musikwissenschaft im späten 19. und frühen 20. Jahrhundert“. Muzikološki Zbornik 50, Nr. 2 (2014): 43-51.

Loos, Helmut. „Heilige Nüchternheit. Der Komponist in der Moderne. Kontinuität statt Bruch“. In Nova glasba v »novi« Evropi med obema svetovnima vojnama - New Music in the "New" Europe Between the Two World Wars, hrsg. von Jernej Weiss, 51-62. Koper, Ljubljana: Založba Univerze na Primorskem, Festival Ljubljana, 2018.

Martienßen, C. A. „Das Institut für Kirchenmusik an der Hochschule für Musik in Leipzig“. Zeitschrift für Musik 89 (1922): 102-105.

Nagler, Franciscus. „Messe und Musik“. Zeitschrift für Musik 89 (1922): 97-99.

Neue Zeitschrift für Musik 87 (1920): 17.

Niechciol, T. „Die Feier des hundertjährigen Bestehens der 'Staatlichen Akademie für Schul- und Kirchenmusik' in Berlin“. Zeitschrift für Musik 89 (1922): 300-302.

Rathert, Wolfgang. „Kult und Kritik. Aspekte der Bach-Rezeption vor dem Ersten Weltkrieg“" In Bach und die Nachwelt, Bd. 3: 190o-1950, hrsg. von Michael Heinemann und Hans-Joachim Hinrichsen, 23-61. Laaber: Laaber Verlag, 2000.

Robert, J. E. „Bach, Beethoven und Stinkbomben“. Zeitschrift für Musik 90 (1923): 6.

Schulze, Hans-Joachim, Ulrich Leisinger und Peter Wollny, Hrsg. Passionsmusiken im Umfeld Johann Sebastian Bachs. Bach unter den Diktaturen 1933-1945 und 1945-1989. Bericht über die Wissenschaftliche Konferenz anlässlich des 69. Bach-Festes der Neuen Bachgesellschaft Leipzig, 29. und 30. März 1994. Hildesheim, Zürich und New York: Olms, 1995.

Wasserloos, Yvonne. Das Leipziger Konservatorium der Musik im 19. Jahrhundert. Anziehung und Ausstrahlungskraft eines musikpädagogischen Modells auf das internationale Musikleben. Hildesheim, Zürich und New York: Olms, 2004.

Wehnert, Martin, Hansachim Schiller und Johannes Forner, Hrsg. Hochschule für Musik Leipzig gegründet als Conservatorium der Musik 1843-1968. Leipzig: Hochschule für Musik, 1968.

Zeitschrift für Musik 88 (1921): 495.

Zeitschrift für Musik 89 (1922): 100, 120, 434. 